\title{
NR2A- and NR2B-containing NMDA receptors in the prelimbic medial prefrontal cortex differentially mediate trace, delay, and contextual fear conditioning
}

\author{
Marieke R. Gilmartin, ${ }^{1}$ Janine L. Kwapis, and Fred J. Helmstetter \\ Department of Psychology, University of Wisconsin-Milwaukee, Milwaukee, Wisconsin 53211, USA
}

\begin{abstract}
Activation of $\mathrm{N}$-methyl-D-aspartate receptors (NMDAR) in the prelimbic medial prefrontal cortex (PL mPFC) is necessary for the acquisition of both trace and contextual fear memories, but it is not known how specific NR2 subunits support each association. The NR2B subunit confers unique properties to the NMDAR and may differentially regulate these two fear memories. Here we show that NR2A-containing NMDARs mediate trace, delay, and contextual fear memories, but NR2Bcontaining NMDARs are required only for trace conditioning, consistent with a role for PL mPFC in working memory.
\end{abstract}

The medial prefrontal cortex (mPFC) is important for the formation and regulation of fear memories. For example, the prelimbic (PL) area of the mPFC is necessary for the acquisition of trace fear conditioning, in which a neutral conditional stimulus (CS) and an aversive unconditional stimulus (UCS) are separated by a stimulus-free "trace" interval of several seconds. Pharmacological inactivation of PL with the $\mathrm{GABA}_{\mathrm{A}}$ agonist muscimol or blockade of the MAPK signaling pathway with U0126 prior to training impairs the formation of this memory (Runyan et al. 2004; Gilmartin and Helmstetter 2010; Guimarais et al. 2011). In contrast, PL is not necessary for standard delay conditioning, in which the CS and UCS are not separated in time (Morgan and LeDoux 1995; Quirk et al. 2000; Corcoran and Quirk 2007; Gilmartin and Helmstetter 2010). These findings lend support to the idea that PL mPFC may serve to bridge the trace interval during trace fear conditioning, providing a representation of the CS for association with the UCS (Baeg et al. 2001; Knight et al. 2004; Gilmartin and McEchron $2005 \mathrm{~b})$. The contribution of PL is not limited, however, to trace conditioning, and is also necessary for contextual fear conditioning acquired simultaneously with either trace or delay conditioning (Gilmartin and Helmstetter 2010). Little is known about the molecular mechanisms supporting fear memory formation in mPFC and whether different processes mediate trace and contextual fear memory. We have previously shown that NMDA receptor activation is necessary for both trace and background contextual fear conditioning (Gilmartin and Helmstetter 2010). NMDA receptors are heteromeric complexes of typically two NR1 and two NR2 subunits, and the specific NR2 subunit confers unique properties to the NMDA receptor (Cull-Candy et al. 2001). The NR1/NR2B complexes have a slow deactivation on the order of seconds, which is amenable to temporal associations or persistent firing characteristics of recurrent circuits (Wang 1999; Wang et al. 2008). In contrast, NR1/NR2A complexes have a rapid deactivation, and represent the predominant NMDAR complex at most mature synapses in the forebrain (Cull-Candy et al. 2001). NR2A-containing receptors may have a more general role in synaptic activity when compared with NR2B-containing receptors (Walker and Davis 2008). Thus, we predict that NR2A-containing receptors in PL are necessary for trace and contextual associations and NR2B-containing receptors are necessary only for trace conditioning. Support for this

\footnotetext{
${ }^{1}$ Corresponding author
}

E-mail marieke.gilmartin@gmail.com

Article is online at http://www.learnmem.org/cgi/doi/10.1101/Im.030510.113. hypothesis comes from a recent study by Radulovic and colleagues showing that NR2B and NR2A subunits differentially regulate trace and contextual fear conditioning in the dorsal hippocampus (Gao et al. 2010). NR2A-containing NMDARs are necessary for both trace and contextual memory, but NR2B-containing receptor activation specifically regulates trace conditioning. However, the mechanisms supporting memory formation in mPFC may be distinct from those observed in the hippocampus. Zhuo and colleagues found that hippocampal NR2B-containing NMDARs are not necessary for contextual fear conditioning, but in anterior cingulate cortex (ACC), blockade of NR2B impaired contextual fear memory (Zhao et al. 2005). Furthermore, in the amygdala, injection of an NR2B antagonist impaired the formation of both delay and contextual fear conditioning (Rodrigues et al. 2001; Walker and Davis 2008). These findings suggest that the contribution of NMDAR subunits to CS and contextual fear memory is regionspecific. To better understand memory-related plasticity in prefrontal cortex it is important to determine the roles of PL NR2 subunits in trace and contextual fear conditioning. Here we examined the contribution of NR2B- and NR2A-containing NMDARs to the formation of trace and contextual memory in PL using pretraining injections of the specific NR2B antagonist Ro25-6981 or the NR2A-preferring antagonist NVP-AAM077.

Fifty-eight adult male Long-Evans rats (325-400 g, Harlan, IN) were housed individually and received food and water ad libitum. All procedures were approved by the Institutional Animal Care and Use Committee and were in accordance with the NIH Guidelines for the Care and Use of Experimental Animals. Under isoflurane anesthesia in 100\% oxygen (induction, 4\%; maintenance, $1 \%-2 \%$ ), guide cannulae (26 ga) were stereotaxically lowered bilaterally in PL at a $15^{\circ}$ angle to vertical: AP $+3.2 \mathrm{~mm}$, ML $\pm 1.6 \mathrm{~mm}$, DV $-3.2 \mathrm{~mm}$ from the skull. Cannulae were secured to the skull with dental acrylic, and 33-ga dummy cannulae were screwed into the guide cannulae to prevent clogging. Following recovery from surgery (12-15 d), rats received $3 \mathrm{~d}$ of acclimation to transport and gentle restraint for microinjections as previously described (Gilmartin and Helmstetter 2010). On the third acclimation day, rats received mock injections, in which the dummy cannulae were removed and the injection cannulae were briefly lowered to the PL, but no injections were delivered. On the day of conditioning, rats received microinjections $(0.3 \mu \mathrm{L} /$ hemisphere at a rate of $0.5 \mu \mathrm{L} / \mathrm{min}$ ) of the specific antagonist of NR2B-containing NMDARs, Ro25-6981 (2 $\mu \mathrm{g} / \mu \mathrm{L}, \mathrm{R} 7150$ Sigma-Aldrich), the NR2A-preferring antagonist, NVP-AAM077 $(1 \mu \mathrm{g} / \mu \mathrm{L}, \mathrm{P} 1999$ 


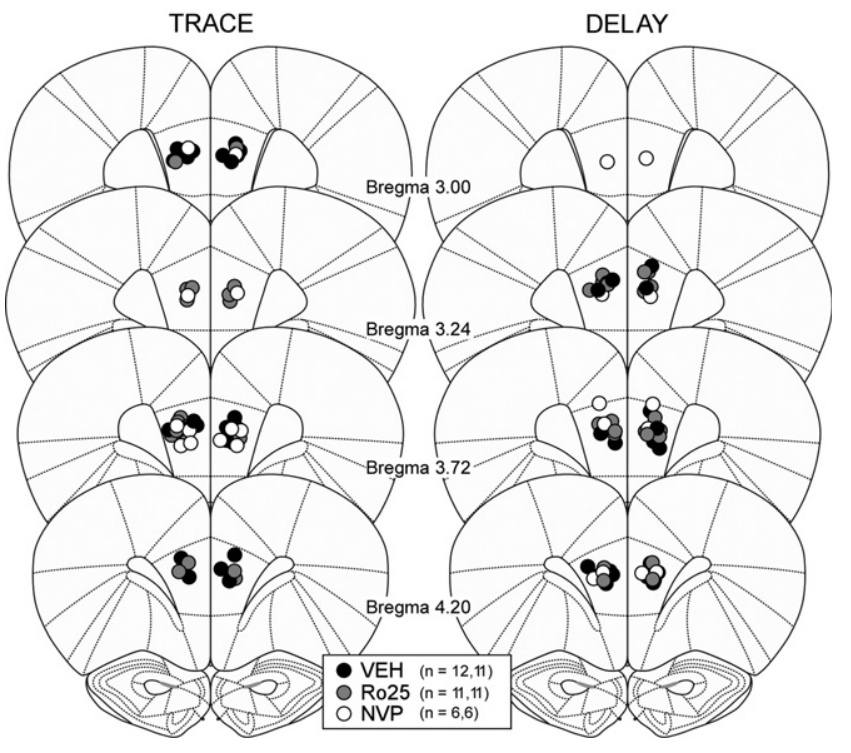

Figure 1. Placement of injector tips bilaterally in PL mPFC. Diagrams were adapted from Paxinos and Watson 2007 (with permission from Elsevier (c) 2007). The number of rats in each group (trace, delay) is shown next to each drug.

Sigma-Aldrich), or vehicle (10\% DMSO in dH2O) 15 min prior to training (see Fig. 1 for the number of subjects in each of the six groups). These doses were chosen based on effective doses in vivo in hippocampus, amygdala, and ACC (Zhao et al. 2005; Walker and Davis 2008; Gao et al. 2010).

Conditioning occurred in a set of four Plexiglas and stainlesssteel conditioning chambers, each housed in a sound-attenuating outer chamber and illuminated with a white incandescent house lamp (see Gilmartin et al. 2012 for specific details of the conditioning and testing apparatus). After a 6-min baseline period, rats received six trace conditioning trials or four delay conditioning trials, in which a 10 -sec white noise CS $(72 \mathrm{~dB})$ was paired with a 1-sec footshock UCS (1 mA). For trace fear conditioning, the CS offset and UCS onset were separated by an empty 20 -sec trace interval and the intertrial interval (ITI) for this session was $240 \pm 20 \mathrm{sec}$ (total session duration, $33 \mathrm{~min}$ ). For delay conditioning, the UCS was delivered at the offset of the CS and the ITI was $110 \pm 20 \mathrm{sec}$ (total session duration, $17 \mathrm{~min}$ ). Six-trial trace conditioning and four-trial delay conditioning produce similar levels of conditional fear to the CS (Kwapis et al. 2011). During training, rats learned to associate both the auditory CS and the training context with the occurrence of the UCS. One rat in the Trace Ro25 group failed to respond to half of the UCS presentations during training and was excluded from analyses. The following day, rats were tested drug-free for memory of each association. Contextual fear memory was assessed by measuring conditional freezing during re-exposure to the original training chamber. Conditional freezing to the CS was tested $2-3 \mathrm{~h}$ later in a novel testing chamber in a separate room and differed from the training chamber in illumination, texture, and odor. The CS retention test consisted of a 2-min baseline period followed by eight 30-sec CS presentations (ITI $60 \mathrm{sec}$ ).

Freezing was defined as the cessation of all movement except that needed for respiration and was used as the measure of conditional fear during all training and testing sessions (Fanselow and Bolles 1979). Freezing was scored automatically in real-time using FreezeScan 1.0 detection software (Clever Sys, Inc.). All statistical analyses were performed with Statistica version 9 (Statsoft, Inc.).
Each training group was analyzed separately and differences in freezing between each drug and vehicle controls were analyzed using one-way ANOVAs (context retention) or mixed model ANOVAs (acquisition, CS retention), which included the following factors: a repeated measure of Period (for acquisition: Baseline, Trial; for CS retention: Baseline, CS1-4) and a between factor of Drug. The average of the first four CS presentations was analyzed to avoid extinction-related CS freezing. Fisher LSD post-hoc tests were used to make pairwise comparisons on significant main effects and interactions. An $\alpha$ level of 0.05 was required for significance in all analyses.

At the end of the experiment, rats were deeply anesthetized with $5 \%$ isoflurane, transcardially perfused with $0.9 \%$ saline followed by $10 \%$ buffered formalin. After cryoprotection with $20 \%$ sucrose/formalin, brains were frozen, sectioned coronally, mounted on glass slides, and stained with Cresyl violet. All rats had accurate cannula placement in the PL of the mPFC (Fig. 1).

Pre-training drug infusion did not impair the acquisition of conditional freezing responses during training. Figure 2 shows the freezing during each pairing for all groups (90-sec period starting at CS onset). Rats injected with the NR2B antagonist Ro25 prior to trace conditioning showed similar acquisition to vehicle (VEH) control rats (Fig. 2A). Analysis of freezing across trials revealed a significant effect of Trial, $F_{(6,126)}=27.286, P<0.0001$, but no main effect of Drug or Drug $\times$ Trial interaction $\left(P^{\prime} s>0.05\right)$. Rats injected with the NR2A antagonist NVP prior to trace conditioning appeared to show reduced freezing on some trials compared with VEH controls, but this reduction was not statistically reliable. Analysis of freezing revealed a significant effect of Trial, $F_{(6,96)}=$ 13.506, $P<0.0001$, but no main effect of Drug or Drug $\times$ Trial interaction $\left(P^{\prime} \mathrm{s}>0.05\right)$. Similarly for delay conditioning, neither drug impaired the acquisition of conditional freezing $\left(P^{\prime} \mathrm{s}>\right.$ 0.05) (Fig. 2B). Rats in each drug and training condition also exhibited similar post-shock freezing $(60 \mathrm{sec})$ across trials (data not shown, all $\left.P^{\prime} \mathrm{s}>0.05\right)$, indicating similar shock reactivity in each drug group. Because CS freezing is not independent of contextual freezing during the training session, we examined the acquisition of contextual freezing in the $30 \mathrm{sec}$ preceding each CS (data not

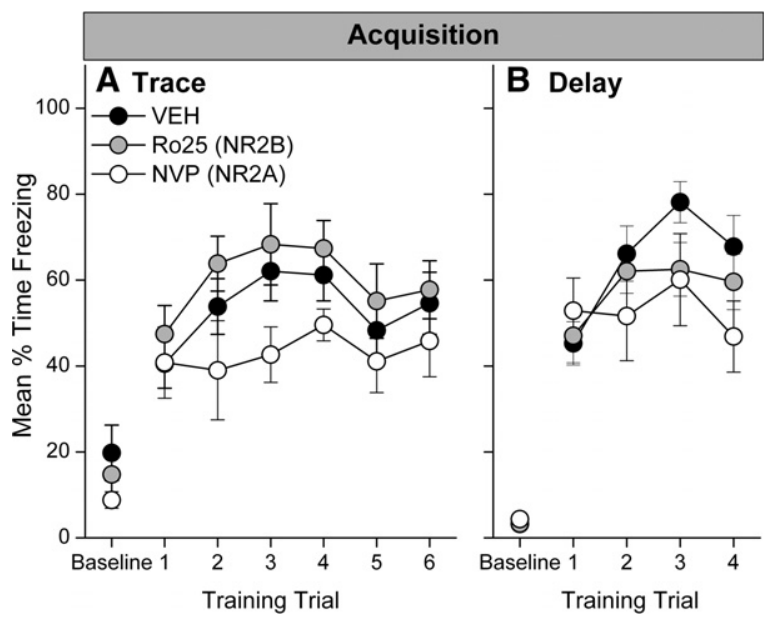

Figure 2. Acquisition of conditional fear. All rats acquired conditional freezing during training. Graphs show the mean percent time each group spent freezing during the training session for trace conditioning $(A)$ or delay conditioning $(B)$. After the 6-min baseline period, each point represents $90 \mathrm{sec}$ starting at CS onset of each trial. Although rats injected with the NR2A antagonist NVP prior to training tended to freeze less in general compared with control rats, this did not represent a significant reduction in freezing $(P>0.10$ for both trace and delay groups). 
shown). No differences were observed across trials between rats injected with the NR2B antagonist and vehicle rats in either trace or delay groups $\left(P^{\prime} \mathrm{s}>0.05\right)$. Rats injected with the NR2A-preferring NMDAR antagonist showed a Drug $\times$ Trial interaction in trace $\left(F_{(5,80)}=2.417, P<0.043\right)$ and delay $\left(F_{(3,45)}=4.083, P<0.012\right)$ groups. Post-hoc analysis revealed that trace NVP rats froze significantly less than controls in the $30 \mathrm{sec}$ before the fourth trial $(P<0.05)$. Delay NVP rats froze significantly more than controls in the $30 \mathrm{sec}$ before the second trial $(P<0.05)$. No differences were observed on any other trial. This suggests that the NR2A antagonist NVP may have a subtle effect on contextual learning during training.

Rats were tested for retention of conditional fear to the CS and context the following day. The NR2A-preferring antagonist NVP administered before training impaired both CS and contextual fear memory at test in trace conditioned rats (Fig. 3A,B). Analysis of freezing during baseline and the mean of the first four CSs revealed a significant Drug $\times$ Period interaction, $F_{(1,16)}=11.282$, $P=0.0040$. Post-hoc analyses showed that NVP rats froze significantly less during the CS compared with controls $(P<0.05)$. NVP rats also froze significantly less than controls during the con-
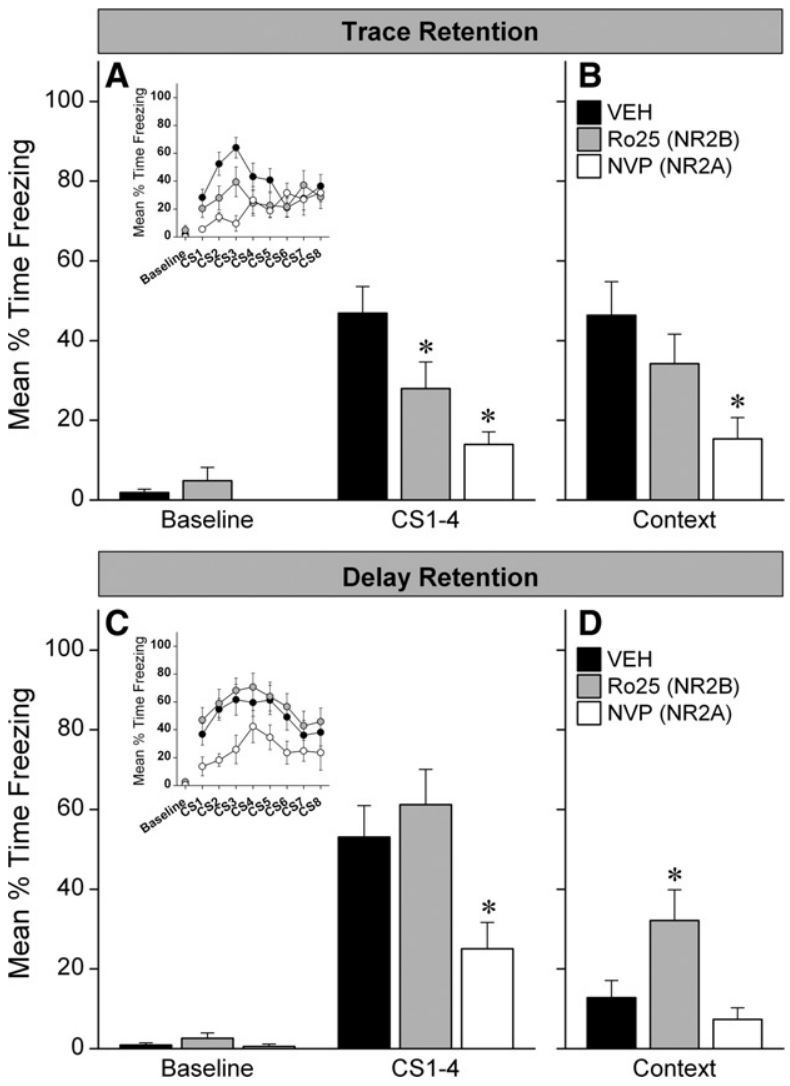

Figure 3. Selective role for NR2B-containing NMDA receptors trace fear conditioning. Graphs show the mean percent time each group spent freezing during the $C S$ retention test $(A, C)$ and context retention test $(B, D)$ for trace and delay rats. Bars represent the average freezing during the 2 min baseline and the average freezing during the first four 30 -sec CS presentations. The freezing during each CS is shown in the insets. While NR2A-containing NMDA receptors are necessary for both tone and contextual memory in trace fear conditioning, NR2B-containing NMDA receptors are important for CS, but not for contextual fear memory. NR2A-containing NMDA receptors may also contribute to stimulus salience processing by $\mathrm{PL}$, as blocking these receptors impairs delay conditioning. $\left.{ }^{*}\right) P<0.05$ relative to VEH. text test, $F_{(1,16)}=6.086, P=0.0253$. In contrast to the NR2A antagonist, pre-training injection of the NR2B antagonist Ro25 impaired $\mathrm{CS}$ but not contextual memory in trace rats. Analysis of the CS test revealed a significant Drug $\times$ Period interaction, $F_{(1,16)}=7.095$, $P=0.0145$, and post-hoc analysis showed that Ro25 rats froze less than controls during the CS $(P<0.05)$. Contextual fear responses were intact in Ro25 rats $(F<1.164, P>0.05)$. These results suggest a differential role in trace CS and contextual learning by NMDARs based on their subunit composition.

Although we have previously shown that delay fear conditioning is unaffected by pre-training blockade of prefrontal NMDARs with APV (Gilmartin and Helmstetter 2010), pretraining injection of the NR2A-preferring antagonist NVP impaired CS memory in delay rats (Fig. 3C). Analysis of freezing during the CS test revealed a significant Drug $\times$ Period interaction, $F_{(1,15)}=5.464, P=0.0337$. Post-hoc analyses showed that NVP rats froze significantly less during the CS compared with controls $(P<0.05)$. Injection of the NR2B antagonist Ro25 in PL had no effect on delay fear conditioning to the CS $(P>0.05)$. Vehicle controls showed unexpectedly low levels of context freezing at test compared with typical responses using this four-trial procedure in previous reports (e.g., Kwapis et al. 2009, 2011). NVP rats exhibited less freezing than controls, but a potential floor effect may have obscured an impairment. Ro25 rats showed increased contextual freezing relative to controls, $F_{(1,20)}=4.862$, $P<0.0393$, which could reflect a role for NR2B in background contextual associations. However, we did not observe enhanced contextual fear by Ro25 in the trace conditioned animals, which suggests to us that the difference in freezing between the VEH and Ro25 delay groups is driven by the low freezing in the controls rather than by specific inhibition of the NR2B subunit. The reason for impaired memory after NVP but not after APV is not clear; however, differential effects by subunit-selective antagonists vs. $\mathrm{APV}$ are not unheard of in other systems. Long-term depression in CA1 cultures was blocked by the NR2B antagonist ifenprodil or Ro25, but not by NVP or APV, despite similar reductions of NMDAR-mediated EPSCs by these inhibitors (Izumi et al. 2006). Whether such differences in that study or in our findings relate to subunit affinity or other factors remains to be determined.

This study provides new information about the contribution of PL mPFC to the formation of fear memory. We have previously shown that trace CS and contextual fear memories require NMDAR activation in PL (Gilmartin and Helmstetter 2010). Here we show that NMDARs containing the NR2A subunit are necessary for both cued and contextual fear conditioning, but NR2B-containing NMDARs are necessary for the association of the CS and UCS across an empty trace interval. This requirement in trace fear conditioning may reflect a role for NR1/NR2B complexes in working memory, a hallmark of prefrontal function (Fuster 2000; Kesner and Churchwell 2011). The NR2B subunit confers a much longer deactivation window to the NMDAR (Monyer et al. 1994; Cull-Candy and Leszkiewicz 2004), which has been shown in computational models of prefrontal circuits to support recurrent persistent activity (Wang 1999; Compte et al. 2000). In vitro, NMDAR-EPSCs at layer $\mathrm{V}$ recurrent synapses in $\mathrm{MPFC}$ exhibited a slower decay and better temporal summation in response to a $20-\mathrm{Hz}$ stimulation compared with visual cortex (Wang et al. 2008). Interestingly, mPFC retains a higher proportion of NR2B subunits in adulthood, compared with that in other cortical regions (Wang et al. 2008). The NR2B subunit is prevalent in early development but shortly after birth it is largely replaced with NR2A subunits (Cull-Candy and Leszkiewicz 2004; Wang et al. 2008). NR2B overexpression in mouse forebrain enhanced prefrontal LTP and improved working memory performance on a number of tasks (Cui et al. 2011). While NR2B-containing NMDARs have been shown to be important for a number of different learning paradigms in different brain 
regions, the selective role for NR2B in trace CS, but not contextual or delay fear, is consistent with a bridging role for PL mPFC in trace conditioning.

The pattern of results in this study is similar to that seen in the hippocampus (Gao et al. 2010). Blockade of NR2B-containing NMDARs in hippocampus impaired trace CS but not contextual fear memory. Although hippocampal neurons do not exhibit persistent firing during the trace interval (Gilmartin and McEchron 2005a), hippocampus is nonetheless important for working memory (Lee and Kesner 2002) and NR2B involvement may contribute to temporal processing in both hippocampus and PL. In contrast to hippocampus and PL, NR2B receptors in ACC do mediate contextual fear conditioning and are necessary for cingulate LTP (Zhao et al. 2005). NR2B activation in ACC during contextual fear conditioning may represent a structure-specific role for these receptors in pain processing during fear conditioning (Tang et al. 2005; Wu and Zhuo 2009). Examination of the intracellular mechanisms downstream of NR2B and NR2A activation in each structure will thus provide insight into structure-specific mechanisms of memory formation. Along these lines, Gao et al. (2010) found that NR2B-containing receptors in the hippocampus are primarily extrasynaptic, whereas NR2A-containing receptors are primarily synaptic, and each subunit uniquely regulated MAPK signaling during trace conditioning. Extrasynaptic localization of NR2Bcontaining receptors is typical of many forebrain structures after NR2B-containing NMDARs at the synapse are replaced by NR2Acontaining NMDARs in early development. As mentioned earlier, NR2B-containing NMDARs remain proportionately high in mPFC relative to other cortical regions (Wang et al. 2008), but the distribution of these receptors at the synapse is not known. This leaves open the possibility that these receptors mediate trace conditioning by different downstream mechanisms in hippocampus and PL. Further work will be needed to address this question. It is important to note that the relative affinity of NVP-AAM077 for the NR2A subunit over the NR2B subunit is not as selective as originally reported (Auberson et al. 2002; Frizelle et al. 2006), and we cannot rule out that some of the effects of NVP-AAM077 on trace CS memory are NR2B-mediated. The recent identification of a new noncompetitive NR2A-selective antagonist TCN201 may be useful in addressing this issue as it continues to be further characterized (Edman et al. 2012). Nonetheless, NVP-AAM007 impaired contextual fear conditioning while the potent NR2B inhibitor did not, suggesting that contextual fear conditioning is primarily mediated by NR2A-containing receptors.

An unexpected result of this study is the impairment in delay conditional fear by injection of the NR2A-preferring antagonist. Lesions or temporary inactivation of PL do not impair delay conditioning, which can be supported by converging CS and UCS inputs in lateral amygdala (Romanski et al. 1993; Morgan and LeDoux 1995; Corcoran and Quirk 2007). Nonetheless, c-fos expression is increased in PL after delay fear conditioning (Furlong et al. 2010) and PL units display learning-related plasticity during training (Garcia et al. 1999; Baeg et al. 2001; Laviolette et al. 2005). More recently, PL has been shown to regulate learning conditional fear to a new stimulus in a blocking paradigm (Furlong et al. 2010). This suggests that delay CS conditional fear can be acquired in the absence of $\mathrm{mPFC}$, but that the $\mathrm{mPFC}$ may normally regulate some aspect of the association during memory formation. In support, disruption of cannabinoid or dopaminergic signaling in $\mathrm{mPFC}$ impairs olfactory delay fear conditional responses and selectively affects the neuronal responses of amygdala-responsive prefrontal units (Laviolette et al. 2005; Laviolette and Grace 2006). Prefrontal activity regulated by the basolateral amygdala may reflect how well the CS predicts the UCS (Garcia et al. 1999). Amygdala input to mPFC suppresses prefrontal firing, possibly via glutamatergic action at cortical parvalbumin-containing inter- neurons (Garcia et al. 1999; Gabbott et al. 2006). NR2A-containing NMDARs may mediate these signaling pathways. These interneurons in mPFC express proportionately more NR2A-containing NMDARs than principal cells (Wang and Gao 2009). Thus the effect of the NR2A antagonist on delay conditioning may represent a disruption in amygdala-PL communication during conditioning, although this remains to be determined. Together, these lines of evidence support a role for PL in modulating delay fear conditioning.

In conclusion, this study shows that NR2A-containing NMDARs are important for both auditory cued and contextual fear memory formation, suggesting that these receptors are part of a common mechanism in PL-mediated memory formation and may contribute to encoding the relative predictability of the CS and contextual stimuli during fear conditioning. NR2Bcontaining NMDARs are selective to trace conditioning and may contribute to a working-memory bridging process across the trace interval in memory formation. The differential contribution of PL NR2A and NR2B subunits in the formation of trace and contextual fear memory resembles that observed in the hippocampus and is distinct from that in anterior cingulate, where NR2B-containing receptors do participate in contextual fear conditioning. These results suggest that NR2B-containing NMDARs participate in memory formation in a structure-specific way and highlight the functional distinction of mPFC subregions. Examination of intracellular signaling downstream of NMDAR activation in mPFC will provide further insight into fear memory formation and regulation in prefrontal circuits.

\section{Acknowledgments}

This research was supported by the National Institute of Mental Health (NIMH) grant R01MH069558 to F.J.H. We thank Erin Schraufnagel for assistance with histology.

\section{References}

Auberson YP, Allgeier H, Bischoff S, Lingenhoehl K, Moretti R, Schmutz M. 2002. 5-Phosphonomethylquinoxalinediones as competitive NMDA receptor antagonists with a preference for the human $1 \mathrm{~A} / 2 \mathrm{~A}$, rather than 1A/2B receptor composition. Bioorg Med Chem Lett 12: 1099-1102.

Baeg EH, Kim YB, Jang J, Kim HT, Mook-Jung I, Jung MW. 2001. Fast spiking and regular spiking neural correlates of fear conditioning in the medial prefrontal cortex of the rat. Cereb Cortex 11: 441-451.

Compte A, Brunel N, Goldman-Rakic PS, Wang XJ. 2000. Synaptic mechanisms and network dynamics underlying spatial working memory in a cortical network model. Cereb Cortex 10: 910-923.

Corcoran KA, Quirk GJ. 2007. Activity in prelimbic cortex is necessary for the expression of learned, but not innate, fears. I Neurosci 27: 840-844.

Cui Y, Jin J, Zhang X, Xu H, Yang L, Du D, Zeng Q, Tsien JZ, Yu H, Cao X. 2011. Forebrain NR2B overexpression facilitating the prefrontal cortex long-term potentiation and enhancing working memory function in mice. PLoS One 6: e20312.

Cull-Candy SG, Leszkiewicz DN. 2004. Role of distinct NMDA receptor subtypes at central synapses. Sci STKE 2004: re16.

Cull-Candy SG, Brickley S, Farrant M. 2001. NMDA receptor subunits: Diversity, development and disease. Curr Opin Neurobiol 11: 327-335.

Edman S, McKay S, Macdonald LJ, Samadi M, Livesey MR, Hardingham GE, Wyllie DJ. 2012. TCN 201 selectively blocks GluN2A-containing NMDARs in a GluN1 co-agonist dependent but non-competitive manner. Neuropharmacology 63: 441-449.

Fanselow MS, Bolles RC. 1979. Naloxone and shock-elicited freezing in the rat. J Comp Physiol Psychol 93: 736-744.

Frizelle PA, Chen PE, Wyllie DJ. 2006. Equilibrium constants for (R)-[(S)-1(4-bromo-phenyl)-ethylamino]-(2,3-dioxo-1,2,3,4tetrahydroquinoxalin-5-yl)-methyl]-phosphonic acid (NVP-AAM077) acting at recombinant NR1/NR2A and NR1/NR2B

$\mathrm{N}$-methyl-D-aspartate receptors: Implications for studies of synaptic transmission. Mol Pharmacol 70: 1022-1032.

Furlong TM, Cole S, Hamlin AS, McNally GP. 2010. The role of prefrontal cortex in predictive fear learning. Behav Neurosci 124: 574-586.

Fuster JM. 2000. Executive frontal functions. Exp Brain Res 133: 66-70. 
Gabbott PL, Warner TA, Busby SJ. 2006. Amygdala input monosynaptically innervates parvalbumin immunoreactive local circuit neurons in rat medial prefrontal cortex. Neuroscience 139: 1039-1048.

Gao C, Gill MB, Tronson NC, Guedea AL, Guzman YF, Huh KH, Corcoran KA, Swanson GT, Radulovic J. 2010. Hippocampal NMDA receptor subunits differentially regulate fear memory formation and neuronal signal propagation. Hippocampus 20: 1072-1082.

Garcia R, Vouimba RM, Baudry M, Thompson RF. 1999. The amygdala modulates prefrontal cortex activity relative to conditioned fear. Nature 402: 294-296.

Gilmartin MR, Helmstetter FJ. 2010. Trace and contextual fear conditioning require neural activity and NMDA receptor-dependent transmission in the medial prefrontal cortex. Learn Mem 17: 289-296.

Gilmartin MR, McEchron MD. 2005a. Single neurons in the dentate gyrus and CA1 of the hippocampus exhibit inverse patterns of encoding during trace fear conditioning. Behav Neurosci 119: 164-179.

Gilmartin MR, McEchron MD. 2005b. Single neurons in the medial prefrontal cortex of the rat exhibit tonic and phasic coding during trace fear conditioning. Behav Neurosci 119: 1496-1510.

Gilmartin MR, Kwapis JL, Helmstetter FJ. 2012. Trace and contextual fear conditioning are impaired following unilateral microinjection of muscimol in the ventral hippocampus or amygdala, but not the medial prefrontal cortex. Neurobiol Learn Mem 97: 452-464.

Guimarais M, Gregorio A, Cruz A, Guyon N, Moita MA. 2011. Time determines the neural circuit underlying associative fear learning. Front Behav Neurosci 5: 89.

Izumi Y, Auberson YP, Zorumski CF. 2006. Zinc modulates bidirectional hippocampal plasticity by effects on NMDA receptors. J Neurosci 26: $7181-7188$.

Kesner RP, Churchwell JC. 2011. An analysis of rat prefrontal cortex in mediating executive function. Neurobiol Learn Mem 96: 417-431.

Knight DC, Cheng DT, Smith CN, Stein EA, Helmstetter FJ. 2004. Neural substrates mediating human delay and trace fear conditioning. $J$ Neurosci 24: $218-228$.

Kwapis JL, Jarome TJ, Lonergan ME, Helmstetter FJ. 2009. Protein kinase Mzeta maintains fear memory in the amygdala but not in the hippocampus. Behav Neurosci 123: 844-850.

Kwapis JL, Jarome TJ, Schiff JC, Helmstetter FJ. 2011. Memory consolidation in both trace and delay fear conditioning is disrupted by intra-amygdala infusion of the protein synthesis inhibitor anisomycin. Learn Mem 18: 728-732.

Laviolette SR, Grace AA. 2006. Cannabinoids potentiate emotional learning plasticity in neurons of the medial prefrontal cortex through basolateral amygdala inputs. J Neurosci 26: 6458-6468.

Laviolette SR, Lipski WJ, Grace AA. 2005. A subpopulation of neurons in the medial prefrontal cortex encodes emotional learning with burst and frequency codes through a dopamine D4 receptor-dependent basolateral amygdala input. J Neurosci 25: 6066-6075.
Lee I, Kesner RP. 2002. Differential contribution of NMDA receptors in hippocampal subregions to spatial working memory. Nat Neurosci 5: $162-168$.

Monyer H, Burnashev N, Laurie DJ, Sakmann B, Seeburg PH. 1994. Developmental and regional expression in the rat brain and functional properties of four NMDA receptors. Neuron 12: 529-540.

Morgan MA, LeDoux JE. 1995. Differential contribution of dorsal and ventral medial prefrontal cortex to the acquisition and extinction of conditioned fear in rats. Behav Neurosci 109: 681-688.

Paxinos G, Watson C. 2007. The rat brain in stereotaxic coordinates. Academic Press, Amsterdam, The Netherlands.

Quirk GJ, Russo GK, Barron JL, Lebron K. 2000. The role of ventromedial prefrontal cortex in the recovery of extinguished fear. J Neurosci 20: $6225-6231$.

Rodrigues SM, Schafe GE, LeDoux JE. 2001. Intra-amygdala blockade of the NR2B subunit of the NMDA receptor disrupts the acquisition but not the expression of fear conditioning. J Neurosci 21: 6889-6896.

Romanski LM, Clugnet MC, Bordi F, LeDoux JE. 1993. Somatosensory and auditory convergence in the lateral nucleus of the amygdala. Behav Neurosci 107: 444-450.

Runyan JD, Moore AN, Dash PK. 2004. A role for prefrontal cortex in memory storage for trace fear conditioning. J Neurosci $\mathbf{2 4}$ $1288-1295$.

Tang J, Ko S, Ding HK, Qiu CS, Calejesan AA, Zhuo M. 2005. Pavlovian fear memory induced by activation in the anterior cingulate cortex. $\mathrm{Mol}$ Pain 1: 6 .

Walker DL, Davis M. 2008. Amygdala infusions of an NR2B-selective or an NR2A-preferring NMDA receptor antagonist differentially influence fear conditioning and expression in the fear-potentiated startle test. Learn Mem 15: 67-74.

Wang XJ. 1999. Synaptic basis of cortical persistent activity: The importance of NMDA receptors to working memory. J Neurosci 19: 9587-9603.

Wang HX, Gao WJ. 2009. Cell type-specific development of NMDA receptors in the interneurons of rat prefrontal cortex. Neuropsychopharmacology 34: 2028-2040.

Wang H, Stradtman GG III, Wang XJ, Gao WJ. 2008. A specialized NMDA receptor function in layer 5 recurrent microcircuitry of the adult rat prefrontal cortex. Proc Natl Acad Sci 105: 16791-16796.

Wu LJ, Zhuo M. 2009. Targeting the NMDA receptor subunit NR2B for the treatment of neuropathic pain. Neurotherapeutics 6: 693-702.

Zhao MG, Toyoda H, Lee YS, Wu LJ, Ko SW, Zhang XH, Jia Y, Shum F, Xu H, Li BM, et al. 2005. Roles of NMDA NR2B subtype receptor in prefrontal long-term potentiation and contextual fear memory. Neuron 47: $859-872$.

Received January 23, 2013; accepted in revised form February 23, 2013. 


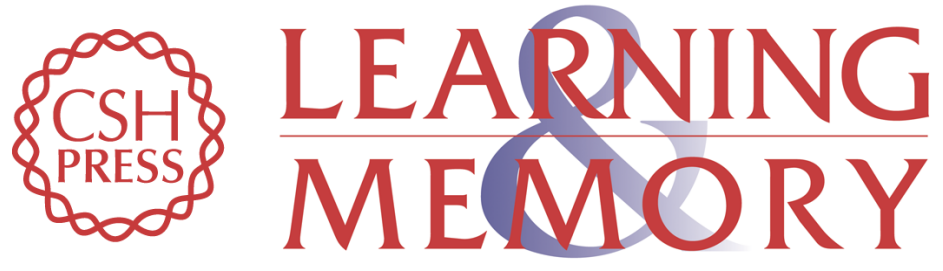

\section{NR2A- and NR2B-containing NMDA receptors in the prelimbic medial prefrontal cortex differentially mediate trace, delay, and contextual fear conditioning}

Marieke R. Gilmartin, Janine L. Kwapis and Fred J. Helmstetter

Learn. Mem. 2013, 20:

Access the most recent version at doi:10.1101//m.030510.113

References This article cites 41 articles, 15 of which can be accessed free at:

http://learnmem.cshlp.org/content/20/6/290.full.html\#ref-list-1

License

Email Alerting

Receive free email alerts when new articles cite this article - sign up in the box at the Service top right corner of the article or click here. 